\title{
Comparison of E Test and Disc Diffusion Methods for Susceptibility Testing of Filamentous Fungi; Experience of a Routine Lab
}

\author{
Reem Mostafa Hassan, ${ }^{1,}{ }^{*}$ Dalia Kadry Ismail, ${ }^{1}$ and Yasmine Samy Elkholy ${ }^{2}$ \\ ${ }^{1}$ Department of Clinical and Chemical Pathology, Faculty of Medicine, Cairo University, Cairo, Egypt \\ ${ }^{2}$ Department of Medical Microbiology and Immunology, Faculty of Medicine, Cairo University, Cairo, Egypt \\ "Corresponding author: Clinical and Chemical Pathology Department, Faculty of Medicine, Cairo University, 1 Al-Saray St., Al-Manial, Cairo 11562, Egypt. Tel: +011-41399472, Fax: \\ +202-23649281, E-mail: reem.mostafa@kasralainy.edu.eg
}

Received 2016 December 11; Revised 2017 July 09; Accepted 2017 September 19.

\begin{abstract}
Background: Testing for antifungal susceptibility to newly introduced drugs is standardized through the clinical laboratory standard institute (CLSI) broth micro-dilution method for testing of molds (M38-A2), yet is difficult to use routinely.

Objectives: To compare two agar-based diffusion methods on two types of media for fungal drug sensitivity testing. Materials and Methods: The E-test method and the disc diffusion method were used on non-supplemented Muller Hinton agar (MHA) and RPMI for evaluating the in-vitro susceptibility of 48 clinical isolates of filamentous fungi to amphotericin B, itraconazole, voriconazole, and caspofungin.

Results: Categorical agreement between E-test and disc diffusion method for itraconazole and voriconazole was 100\%, yet for amphotericin B on MHA agar was $66.67 \%$, and on RPMI, it was $47.92 \%$. The correlation coefficient (R) between the inhibition zone diameters when using MHA and RPMI for itraconazole, voriconazole, amphotericin B and caspofungin was $+0.745,+0.901+0.649$, and +0.409 , respectively.

Conclusions: Routine antifungal susceptibility testing using disk diffusion can be implemented in routine microbiology work up in limited resources.
\end{abstract}

Keywords: Aspergillus, Fungal Drug Sensitivity Tests, Diffusion, Fungi

\section{Background}

Fungal infections are increasing worldwide due to increased incidence of immunocompromised patients either through diseases of the immune system or through the use of immunosuppressive agents (1).

Available antifungal drugs are expanding with introduction of new agents, such as triazoles and echinocandins, with the development of resistance to standard used antifungal drugs, such as amphotericin B (2).

Testing for antifungal susceptibility to these drugs is standardized through the clinical laboratory standard institute (CLSI) broth micro-dilution method for testing of molds (M38-A2) as the reference gold standard procedure, yet is difficult to use routinely in a clinical lab (3).

Various antifungal susceptibility (AFS) testing for filamentous fungi have been proposed, including disk diffusion (DD), E-test, and other commercial tests aimed at standardizing an ideal susceptibility method that is easy, reproducible, accurate, and cost-effective (4). E-test is one of the alternatives yet requires a special media (RPMI 1640 or Ca- sitone agar) and sometimes shows higher MIC values (5).

In 2010, CLSI published a reference method (M51A) for disk diffusion antifungal susceptibility testing of non-dermatophyte filamentous fungi. This document describes guidelines for testing the susceptibility of opportunistic molds to triazoles, amphotericin B, and caspofun$\operatorname{gin}(6)$.

In this study, two agar-based diffusion methods were compared, the E-test method and the disc diffusion method, for evaluating the in vitro susceptibility of 48 clinical isolates of filamentous fungi, including Aspergillus spp. and Fusarium spp., other molds to amphotericin B, itraconazole, voriconazole and caspofungin. Disc diffusion method was performed on two media for comparison; non-supplemented Muller Hinton agar (MHA) and RPMI.

The agreement between E-test minimum inhibitory concentrations (MIC) and zone diameters of categorical susceptibility as proposed by CLSI document M51-A 2010, was determined. 


\section{Methods:}

\subsection{Fungal Isolates}

Forty-eight filamentous fungi isolates were collected from different clinical samples of patients, admitted to Kasr Al-Ainy Hospital, a tertiary care academic medical hospital of Cairo University, Egypt. An informed written consent was obtained from each patient admitted to the hospital and participating in the study. Clinical pathology department, faculty of medicine, and Cairo University ethical committee approved the research, as it was performed on routine samples provided to the department lab. No extra samples were withdrawn for the sake of the research.

\subsection{Susceptibility Testing and Breakpoints}

Both E-test and disc diffusion susceptibility testing were done for isolates according to CLSI guidelines and as described before $(6,7)$.

Isolates were cultured on Sabouraud Dextrose agar (SDA) for five to seven days at $35^{\circ} \mathrm{C}$ to obtain adequate amounts of spores; the conidia of the isolates were mixed in sterile saline with two drops of $0.05 \%$ tween 20 . The turbidity of the mixture was adjusted spectrophotometrically to optical density range of 0.09 to 0.11 at $530 \mathrm{~nm}$ (equivalent to106 spores $/ \mathrm{mL}$ ).

\subsection{E-Test (for Amphotericin B, Itraconazole, Voriconazole and Caspofungin)}

RPMI 1640, supplemented with 1.5\% agar and 2\% glucose and buffered to $\mathrm{pH} 7.0$ with $0.165 \mathrm{M}$ Morpholine Propane Sulfonic acid (MOPS) buffer (Sigma, St. Louis, MO) was used in 9-cm diameter plates containing RPMI media at a depth of $4.0 \mathrm{~mm}$.

The MIC or minimum effective concentration (MEC) in case of caspofungin only was determined after 24 and 48 hours of incubation at $35^{\circ} \mathrm{C}$. The MIC was determined at the point of significant inhibition of fungal growth, whereas MEC was defined as the lowest concentration leading to aberrant growth. The MIC values were interpreted according to CLSI guidelines (3).

\subsection{Disk Diffusion Method}

The entire surface of each 90-mm non-supplemented MH agar plate and RPMI 1640 plate was inoculated with undiluted inoculum suspension. Disks for itraconazole (10 $\mu \mathrm{g})$, voriconazole $(1 \mu \mathrm{g})$, amphotericin $\mathrm{B}(10 \mu \mathrm{g})$, and caspofungin $(5 \mu \mathrm{g}$ ) were obtained commercially (Liofilchem $\AA$ Italy) and placed on the surface of agar plates, according to CLSI guidelines (6).

After using the discs, the plates were incubated at $35^{\circ} \mathrm{C}$ for 24 hours. Zone diameters were measured to the nearest whole millimeter at a point, in which there was a prominent reduction of growth (80\% for azoles and caspofungin) or no visible growth (100\% inhibition for amphotericin B) (8).

Disc diffusion inhibition zone diameters were categorized to susceptible, intermediate, and resistant according to epidemiologic cut off values proposed elsewhere $(8,9)$. Results were correlated with MIC determined by E-test to determine categorical agreement between both methods. Details are shown in Table 1.

\subsection{Data Analysis}

In the current study, the researchers determined the categorical agreement level between E-test (MIC) and disc diffusion diameters, as opposed to the following disagreement parameters: very major error - resistant parameter (R) in E-test and susceptible (S) in disc diffusion; major error - $S$ by E-test and $\mathrm{R}$ by disc diffusion; minor error - shifts between $S$ and intermediate or intermediate and R. Statistical analysis was performed using SPSS 13.0 for Windows, by linear regression analysis and the computation of Pearson's correlation coefficients between the normalized Log transforms of MIC endpoints and the inhibition zone diameters of the four antifungal agents.

\section{Results}

In the current study, 48 filamentous fungi isolates were collected from different clinical samples, including tissue biopsies 37.5\% $(n=18)$, bronchoalveolar lavage $20.8 \%(n=$ $10)$, ear swab $14.6 \%(n=7)$, pus $12.5 \%(n=6)$, sputum $8.3 \%$ ( $=4$ ), ascitic fluid, synovial fluid, cerebrospinal fluid, each 2.1\% (each $\mathrm{n}=1$ ).

Identification of the species was done according to colony morphology and microscopic examination of conidia. Aspergillus fumigatus accounted for $35.4 \%$ of the isolates, while $31.2 \%$ were Aspergillus flavus, $11.3 \%$ were Aspergillus niger, whereas $10.4 \%$ were Fusarium spp., similarly 4.1\% were Aspergillus terreus, and finally $2.1 \%$ accounted for each of Fonsecaea pedrosoi, Bipolaris spp. and Penicillium spp. However, a single isolate could not be identified at the species level.

Antifungal susceptibility testing results showed that $95.8 \%$ of the isolates were sensitive to itraconazole, and 91.7\% were sensitive to voriconazole. Regarding caspofungin, $98 \%$ of the isolates were sensitive. For amphotericin $\mathrm{B}$, discrepancy was noticed, where sensitivity ranged from $79.1 \%$ to $27.15 \%$ when using different testing methods and media. Details are shown in Table 2.

Categorical agreement for itraconazole between E-test and disc diffusion method using both MHA and RPMI agar 


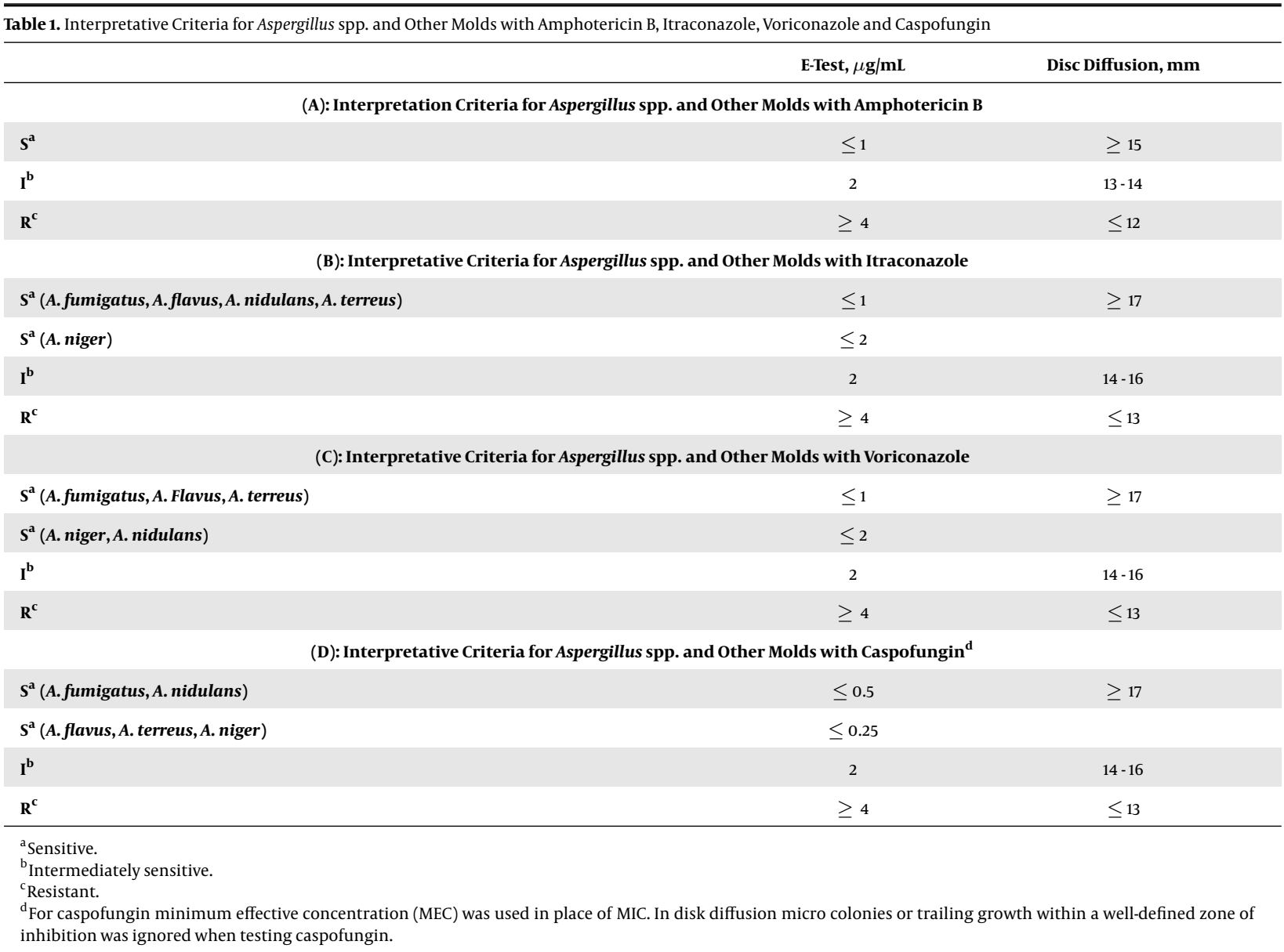

in the current study was $100 \%$. The correlation coefficient (R) between Log2 MICs and the inhibition zone diameters for itraconazole when using MHA was -0.481, and when using RPMI, this was - 0.585 (inversely proportional relation) (Figure $1 \mathrm{~A}-1 \mathrm{~B}$ ).

The correlation coefficient $(R)$ between the inhibition zone diameters for itraconazole when using MHA and RPMI was +0.745 (direct proportion relation). The correlation coefficient $(\mathrm{R})$ between Log2 MICs and the inhibition zone diameters for voriconazole, when using MHA, was -0.310 , and when using RPMI, was -0.319. The correlation coefficient $(R)$ between the inhibition zone diameters for voriconazole, when using MHA and RPMI was +0.901 (Figure 1A - 1C). Categorical agreement result is to be mentioned before results of correlation coefficient. Categorical agreement for voriconazole between E-test and disc diffusion method using both MHA and RPMI agar in the current study was $100 \%$.

The lowest categorical agreement obtained in this study was with amphotericin B; for E-test with disc diffusion on MHA agar, this was $66.67 \%$ with 14 major errors and two minor errors. However, with disc diffusion on RPMI, categorical agreement was $47.92 \%$ with 21 major errors and four minor errors. The correlation coefficient $(R)$ between Log2 MICs and the inhibition zone diameters for amphotericin B when using MHA was -0.599 and when using RPMI was -0.554 . The correlation coefficient $(R)$ between the inhibition zone diameters for amphotericin B when using MHA and RPMI, was +0.649 (Figure 1D).

Categorical agreement for caspofungin between E-test and disc diffusion method using both MHA and RPMI agar in the current study was $100 \%$, with data lacking for a single isolate (i.e. tested isolates were 47). The correlation coefficient $(\mathrm{R})$ between the inhibition zone diameters for caspofungin when using MHA and RPMI was +0.409 (Figure 1E).

In all the above cases, the computed $\mathrm{R}$ values exhibited statistical significance with $\mathrm{P}$ value of $<0.05$

Nevertheless, no correlation coefficient (R) could be obtained between Log2 MICs and the inhibition zone diameters for caspofungin when using MHA or RPMI. 
A

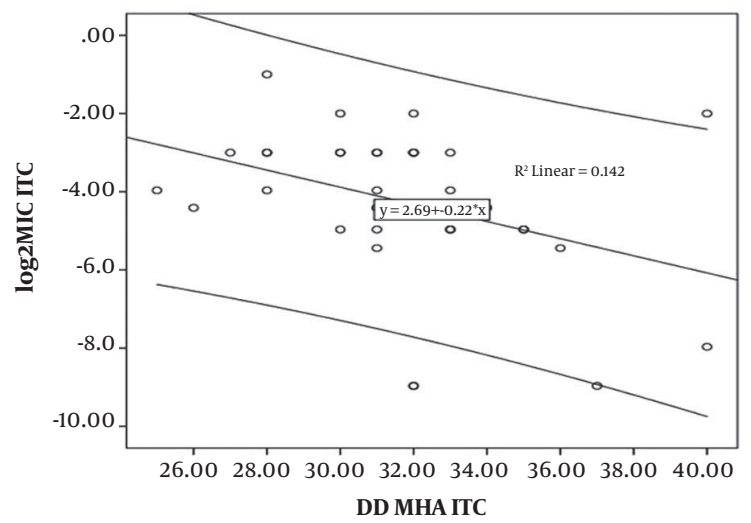

C

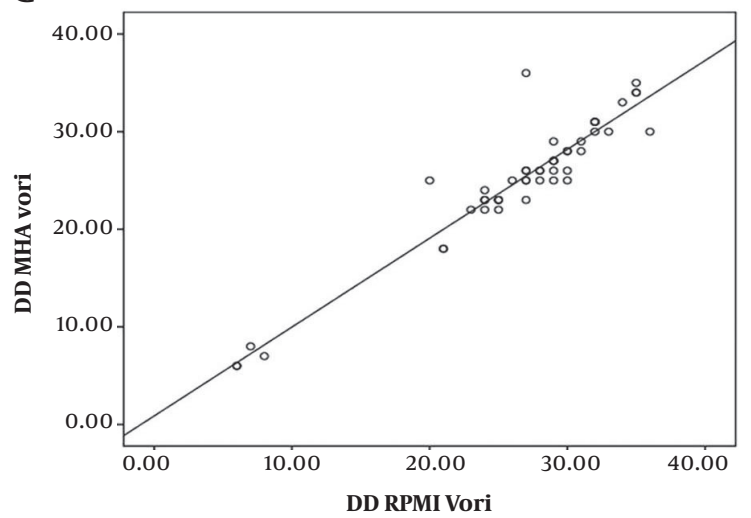

B

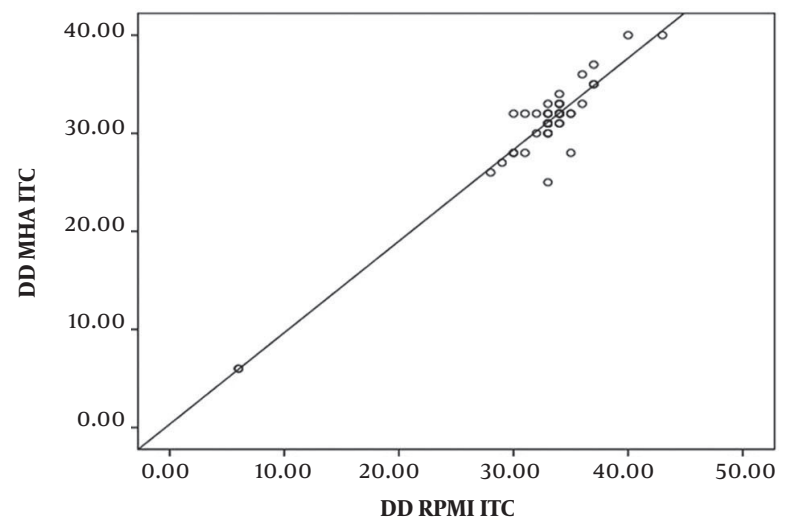

D

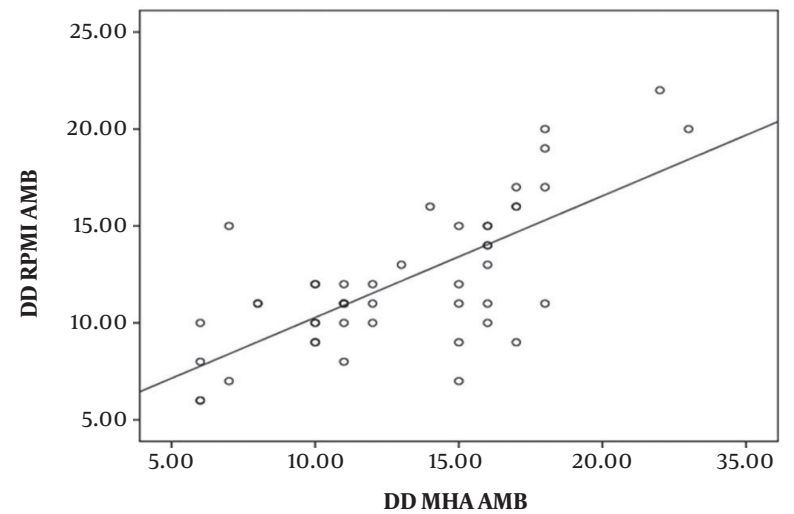

E

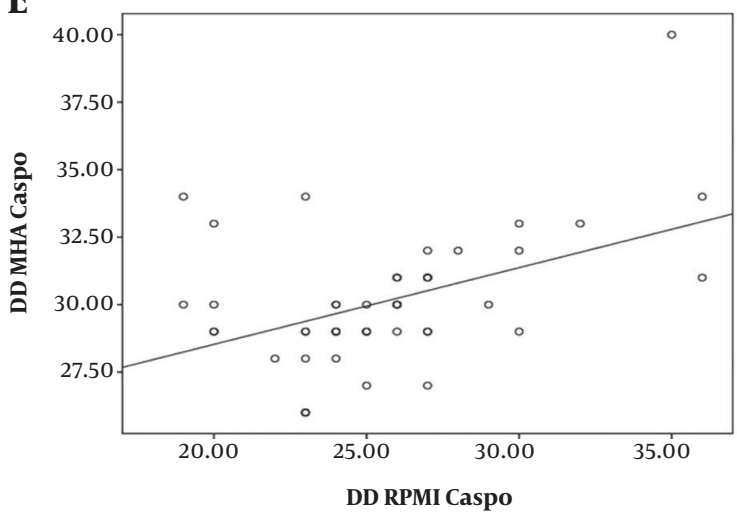

Figure 1. A, The correlation coefficient R between Log2 minimum inhibitory concentration and the inhibition zone diameters for Itraconazole (ITC) using Muller Hinton agar (MHA); B, the correlation coefficient R between inhibition zone diameters on Muller Hinton agar (MHA) and RPMI agar for Itraconazole (ITC); C, the correlation coefficient R between inhibition zone diameters on Muller Hinton agar (MHA) and RPMI agar for Voriconazole (vori); D, the correlation coefficient R between inhibition zone diameters on Muller Hinton agar (MHA) and RPMI agar for Amphotericin B (AMB); E, The correlation coefficient R between inhibition zone diameters on Muller Hinton agar (MHA) and RPMI agar for Caspofungin (caspo).

\section{Discussion}

Availability of a standardized affordable and easy to perform method for antifungal susceptibility testing of fil- amentous fungi is essential, especially for immunocompromised patients (10).

The aim of the present work was to determine a less labor-intensive, cheap and easy to interpret method for 


\begin{tabular}{|c|c|c|}
\hline & Result & Isolates $^{\mathrm{a}}$ \\
\hline \multirow{2}{*}{ E-test Itraconazole } & $S$ & $46(95.8)$ \\
\hline & $\mathrm{R}$ & $2(4.2)$ \\
\hline \multirow{2}{*}{ DD Itraconazole } & $\mathrm{S}$ & $46(95.8)$ \\
\hline & $\mathrm{R}$ & $2(4.2)$ \\
\hline \multirow{2}{*}{ E-test Voriconazole } & $\mathrm{S}$ & $44(91.7)$ \\
\hline & $\mathrm{R}$ & $4(8.3)$ \\
\hline \multirow{2}{*}{ DD Voriconazole } & $\mathrm{S}$ & $44(91.7)$ \\
\hline & $\mathrm{R}$ & $4(8.3)$ \\
\hline \multirow{2}{*}{ E-test Amphotericin B } & $\mathrm{S}$ & $38(79.1)$ \\
\hline & $\mathrm{R}$ & $10(20.8)$ \\
\hline \multirow{3}{*}{ DD Amphotericin B on MHA } & $S$ & $22(45.8)$ \\
\hline & I & $2(4.2)$ \\
\hline & $\mathrm{R}$ & $24(50)$ \\
\hline \multirow{3}{*}{ DD Amphotericin B on RPMI } & S & $13(27.1)$ \\
\hline & I & $4(8.3)$ \\
\hline & $\mathrm{R}$ & $31(64.6)$ \\
\hline \multirow{2}{*}{ E-test Caspofungin } & S & $47(98)$ \\
\hline & $\mathrm{R}$ & $0(0)$ \\
\hline \multirow{2}{*}{ DD Caspofungin } & S & $47(98)$ \\
\hline & $\mathrm{R}$ & $0(0)$ \\
\hline
\end{tabular}

${ }^{\mathrm{a}}$ Values are expressed as No. (\%).

antifungal susceptibility testing through comparison between the two commonly used methods of antifungal susceptibility testing; disc diffusion method and E- test.

Although clinical breakpoints have not been established for mold testing, ECVs are available for Aspergillus spp. versus triazoles and Amphotericin B $(11,12)$. Nevertheless, ECVs can not predict clinical outcome of therapy yet they may aid in detecting isolates with reduced drug susceptibility (non-wild type strains) harboring resistance mutations (13). The wild type strain is defined here as in other studies as the population of organism/MICs in a species-drug combination with no detectable acquired resistance mechanisms $(14,15)$.

Invasive aspergillosis has emerged as the leading cause of morbidity and mortality in immunocompromised patients (16). In the present study, A. fumigatus accounted for $35.4 \%$ of the isolates, followed by A. flavus, that represented $31.2 \%$ of the isolates. Obviously, these two isolates comprised more than $60 \%$ of the filamentous fungi isolated in the current lab. Similarly, many authors reported that A.fumigatus is the most frequently isolated species in case of in- vasive aspergillosis, followed by A. flavus, A. niger, and A. terreus $(17,18)$. In certain hospitals, A. flavus is more common than A. fumigatus, yet, actually the reasons for increased numbers of non-A. fumigatus infections are not fully understood (19).

In the current study, $95.8 \%$ of the isolates were sensitive to itraconazole, $91.7 \%$ were sensitive to voriconazole, and $98 \%$ were sensitive to caspofungin. Categorical agreement obtained in this study for azoles was close to what was reported by Colosi et al. who found 97\% agreement for itraconazole and $96 \%$ for voriconazole and caspofungin (8). However, other studies reported 83\% agreement for itraconazole (9).

Serrano et al. compared a disk diffusion method with E-test procedures in susceptibility tests with voriconazole for 77 isolates of Aspergillus spp. The authors noted an excellent correlation between 24-hour zone sizes and MICs obtained with the E-test (20).

Frequent use of amphotericin B and its lipid formulations continues to increase selection pressure, and thus monitoring of emerging polyene resistance in Aspergillus spp. is important (12). In the current study, sensitivity to amphotericin B was 79.1\% using the E-test and much lower with the disc diffusion method. Similarly, other authors noticed emerging resistance of molds to amphotericin B. One study in Iran also reported that $61.1 \%$ of 108 Aspergillus spp. were resistant to amphotericin B E-test (21). Another study from Tunisia reported that 31 of 37 isolates (83.8\%) of A. flavus isolates obtained from 14 patients with hematological malignancies were resistant $(\geq 2 \mu \mathrm{g} / \mathrm{mL})$ to amphotericin B (22). Gupta et al. found that $31.8 \%$ of 44 Aspergillus spp. had MICs above epidemiologic cut off values (ECVs); denoting potential resistance to amphotericin $B$. The same study pointed also to lower agreement between disc diffusion and the reference microbroth dilution method when using amphotericin $\mathrm{B}$ than voriconazole and caspofungin (87.5\%, 93\%, and $100 \%$ respectively) (4). However, this study was conducted on Aspergillus spp. only (4). Other studies similarly have pointed to such lower agreement with amphotericin B. Colosi et al. reported 76\% agreement between E-test and Neo-Sensitabs tablet diffusion assay on MHA when testing the susceptibility of 100 clinical isolates of filamentous fungi. Errors obtained in that study, were five very major, three major, and 16 minor errors (8).

Closely related to the current findings, several previous studies reported variable high degrees of statistically significant correlations between Log2 MICs and the inhibition zone diameters for itraconazole, voriconazole, and amphotericin B $(8,9,20)$. Similarly, all obtained correlations between disc diffusion zone diameters on MHA and RPMI, were statistically significant in the current study with better results obtained with MHA, which is the stan- 
dard medium recommended according to CLSI (6). However, the only difference noticed in the current study and these other studies was the absence of correlation between Log2 MICs and the inhibition zone diameters for caspofungin when using MHA and RPMI.

To conclude, for disc diffusion method, when comparing non-supplemented MHA and RPMI agar, the correlation coefficient $\mathrm{R}$ between the inhibition zone diameters when using MHA and RPMI for the four tested antifungal agents exhibited statistically significant values. However, the lowest obtained coefficient $\mathrm{R}$ was for amphotericin $\mathrm{B}$, which may highlight the importance of performing such a study on a larger scale to decide which medium will act best for antifungal susceptibility testing. Routine antifungal susceptibility testing using disk diffusion method should be implemented as part of the routine microbiology work up. This method can be easily applied in settings with limited resources.

\section{Footnote}

Declaration of Interests: The authors report no declarations of interest.

\section{References}

1. Marr KA. Fungal infections in oncology patients: update on epidemiology, prevention, and treatment. Curr Opin Oncol. 2010;22(2):138-42. doi: 10.1097/CCO.ob013e328335a755. [PubMed: 20019613].

2. Baddley JW, Marr KA, Andes DR, Walsh TJ, Kauffman CA, Kontoyiannis DP, et al. Patterns of susceptibility of Aspergillus isolates recovered from patients enrolled in the Transplant-Associated Infection Surveillance Network. J Clin Microbiol. 2009;47(10):3271-5. doi: 10.1128/JCM.00854-09. [PubMed: 19692558]. [PubMed Central: PMC2756905].

3. Clinical and Laboratory Standards Institute (formerly NCCLS). Reference Method for Broth Dilution Antifungal Susceptibility Testing of Filamentous Fungi, approved standard. Wayne; 2008.

4. Gupta P, Khare V, Kumar D, Ahmad A, Banerjee G, Singh M. Comparative Evaluation of Disc Diffusion and E-test with Broth Microdilution in Susceptibility testing of Amphotericin B, Voriconazole and Caspofungin against Clinical Aspergillus isolates. J Clin Diagn Res. 2015;9(1):DC04-7. doi: 10.7860/JCDR/2015/10467.5395. [PubMed: 25737984]. [PubMed Central: PMC4347075].

5. Hoffman HL, Pfaller MA. In vitro antifungal susceptibility testing. Pharmacotherapy. 2001;21(8 Pt 2):111S-23S. [PubMed: 11501985].

6. Clinical and Laboratory Standards Institute. Reference method for antifungal disk diffusion susceptibility testing of non-dermatophyte filamentous fungi; approved guideline. Villanova: CLSI document M51-A; 2010.

7. Pfaller MA, Messer SA, Mills K, Bolmstrom A. In vitro susceptibility testing of filamentous fungi: comparison of Etest and reference microdilution methods for determining itraconazole MICs. J Clin Microbiol. 2000;38(9):3359-61. [PubMed: 10970383]. [PubMed Central: PMC87386].

8. Colosi IA, Faure O, Dessaigne B, Bourdon C, Lebeau B, Colosi HA, et al. Susceptibility of 100 filamentous fungi: comparison of two diffusion methods, Neo-Sensitabs and E-test, for amphotericin B, caspofungin, itraconazole, voriconazole and posaconazole. Med My col. 2012;50(4):378-85. doi: 10.3109/13693786.2011.616543. [PubMed: 21981028].
9. Espinel-Ingroff A, Arthington-Skaggs B, Iqbal N, Ellis D, Pfaller MA, Messer S, et al. Multicenter evaluation of a new disk agar diffusion method for susceptibility testing of filamentous fungi with voriconazole, posaconazole, itraconazole, amphotericin B, and caspofungin. $J$ Clin Microbiol. 2007;45(6):1811-20. doi: 10.1128/JCM.00134-07. [PubMed: 17428932]. [PubMed Central: PMC1933077].

10. Lass-Florl C. In vitro susceptibility testing in Aspergillus species: an update. Future Microbiol. 2010;5(5):789-99. doi: 10.2217/fmb.10.34. [PubMed: 20441550].

11. Espinel-Ingroff A, Diekema DJ, Fothergill A, Johnson E, Pelaez T, Pfaller MA, et al. Wild-type MIC distributions and epidemiological cutoff values for the triazoles and six Aspergillus spp. for the CLSI broth microdilution method (M38-A2 document). J Clin Microbiol.2010;48(9):3251-7. doi: 10.1128/JCM.00536-10. [PubMed: 20592159]. [PubMed Central: PMC2937688].

12. Espinel-Ingroff A, Fothergill A, Fuller J, Johnson E, Pelaez T, Turnidge J. Wild-type MIC distributions and epidemiological cutoff values for caspofungin and Aspergillus spp. for the CLSI broth microdilution method (M38-A2 document). Antimicrob Agents Chemother 2011;55(6):2855-9. doi: 10.1128/AAC.01730-10. [PubMed: 21422219]. [PubMed Central: PMC3101428].

13. Espinel-Ingroff A, Canton E, Fothergill A, Ghannoum M, Johnson E, Jones RN, et al. Quality control guidelines for amphotericin B, Itraconazole, posaconazole, and voriconazole disk diffusion susceptibility tests with nonsupplemented Mueller-Hinton Agar (CLSI M51A document) for nondermatophyte Filamentous Fungi. J Clin Microbiol. 2011;49(7):2568-71. doi: 10.1128/JCM.00393-11. [PubMed: 21543581] [PubMed Central: PMC3147863].

14. Diaz-Guerra TM, Mellado E, Cuenca-Estrella M, Rodriguez-Tudela JL. A point mutation in the 14alpha-sterol demethylase gene cyp51A contributes to itraconazole resistance in Aspergillus fumigatus. Antimicrob Agents Chemother. 2003;47(3):1120-4. [PubMed: 12604551]. [PubMed Central: PMC149335].

15. Dalhoff A, Ambrose PG, Mouton JW. A long journey from minimum inhibitory concentration testing to clinically predictive breakpoints: deterministic and probabilistic approaches in deriving breakpoints. Infection. 2009;37(4):296-305. doi: 10.1007/s15010-009-7108-9. [PubMed: 19629383].

16. Richardson M, Lass-Florl C. Changing epidemiology of systemic fungal infections. Clin Microbiol Infect. 2008;14 Suppl 4:5-24. doi: 10.1111/j.1469-0691.2008.01978.x. [PubMed: 18430126].

17. Denning DW. Invasive aspergillosis in immunocompromised patients. Curr Opin Infect Dis. 1994;7(4):456-62. doi: 10.1097/00001432199408000-00007.

18. Lass-Florl C, Griff K, Mayr A, Petzer A, Gastl G, Bonatti H, et al. Epidemiology and outcome of infections due to Aspergillus terreus: 10year single centre experience. $\mathrm{Br} J$ Haematol. 2005;131(2):201-7. doi: 10.1111/j.1365-2141.2005.05763.x. [PubMed: 16197450].

19. Hedayati MT, Pasqualotto AC, Warn PA, Bowyer P, Denning DW. Aspergillus flavus: human pathogen, allergen and mycotoxin producer Microbiology. 2007;153(Pt 6):1677-92. doi: 10.1099/mic.0.2007/0076410. [PubMed: 17526826].

20. Serrano MC, Ramirez M, Morilla D, Valverde A, Chavez M, EspinelIngroff $\mathrm{A}$, et al. A comparative study of the disc diffusion method with the broth microdilution and Etest methods for voriconazole susceptibility testing of Aspergillus spp. J Antimicrob Chemother. 2004;53(5):739-42. doi: 10.1093/jac/dkh172. [PubMed: 15056643].

21. Badiee P, Alborzi A, Moeini M, Haddadi P, Farshad S, Japoni A, et al. Antifungal susceptibility of the aspergillus species by Etest and CLSI reference methods. Arch Iran Med. 2012;15(7):429-32. [PubMed: 22724880].

22. Hadrich I, Makni F, Neji S, Cheikhrouhou F, Bellaaj H, Elloumi $\mathrm{M}$, et al. Amphotericin $\mathrm{B}$ in vitro resistance is associated with fatal Aspergillus flavus infection. Med Mycol. 2012;50(8):829-34. doi 10.3109/13693786.2012.684154. [PubMed: 22587728]. 